\title{
Festschrift für eine Erzähltheorie
}

Franz Karl Stanzel: Die Typischen Erzäh/situationen 1955-2015. Erfolgsgeschichte einer Triade. Würzburg: Königshausen \& Neumann 2015, 351 S.

Dem eigenen theoretischen Entwurf expressis verbis eine Festschrift zu widmen ist sicherlich ein wissenschaftsgeschichtliches Kuriosum. Die Auto-Hommage des Grazer Anglisten und Literaturwissenschaftlers Franz K. Stanzel (Jahrgang 1923) findet ihre Berechtigung in der sechs Jahrzehnte anhaltenden Hartnäckigkeit, mit der ein theoretisches Konzept in seinen Grundzügen verteidigt und durch behutsame Nachjustierungen dem Stand der Forschung diskret angepasst worden ist, belohnt durch eine ebenso lange Anwendungsgeschichte in Forschung und Lehre.

Zur Erinnerung: 1955 veröffentlichte Stanzel seine Habilitationsschrift Die typischen Erzählsituationen im Roman, demonstrierte in Einzel- und Gattungsuntersuchungen die analytische Brauchbarkeit seiner Typologie - unter anderem in der Monographie Typische Formen des Romans von 1964 - und summierte die theoretischen und analytischen Ergebnisse in der Theorie des Erzählens von 1979, die er seitdem in mehreren Neuauflagen, auf Anregungen und Kritik der internationalen Erzählforschung reagierend, im Detail novelliert und kommentiert hat. Vor allem die Konzepte Auktoriale und Personale Erzählsituation, Reflektor- versus Erzählermodus, wie auch die analytische Relevanz der epischen Darstellungsform der Erlebten Rede sind zum festen Bestandteil der Erzähltextanalyse geworden. Dies ist nicht zuletzt dem Umstand zu verdanken, dass die genannten Konzepte nicht immer in Stanzels Sinne benutzt werden und auch in hybride analytische Entwürfe Eingang gefunden haben, etwa in Jochen Vogts Kombination der narratologischen Typologien von Stanzel einerseits und von Gérard Genette, einem seiner prominentesten Kritiker, andererseits. Die "weite Akzeptanz in der lit.wissenschaftlichen Forschung und Lehre « wird mit der »Praktikabilität der an zahlreichen Beispielen aus der Erzähllit. entwickelten Kategorien St.s« erklärt (so im Stanzel-Artikel in A. Nünnings 
Metzler Lexikon Literatur- und Kulturtheorie von 1998). Die Praktikabilität meint freilich nicht die eben erwähnte Möglichkeit der Entlehnung für narratologische Werkzeugkästen, sondern Stanzels genuines Gesamtkonzept, dessen Benutzerfreundlichkeit vor allem auf den Typenkreis und die >Dynamisierung der Erzählsituation` zurückgehen dürfte. Ausgehend von einer phänomenologisch ausgerichteten Rekonstruktion typischer Erzählkonstellationen entwickelte Stanzel ein kreisförmiges Modell, das sowohl eine typologische Gesamtschau aller Möglichkeiten des Erzählens im Hinblick auf drei Grundkategorien, als auch eine für die Interpretation erhellende Ortsbestimmung konkreter Texte oder gar Textpassagen innerhalb des stufenlosen Formkontinuums ermöglicht. Die drei Konstituenten des Kreises (Person, Perspektive, Modus) wurden dem strukturalistischen Hang zu binären Oppositionen insofern angepasst, als ihnen jeweils eine kategoriale Opposition unterlegt wird (Ich-Bezug vs. Er-Bezug; innen vs. außen; Erzähler vs. Reflektor). Trotz kontroverser Diskussion in der Erzählforschung vor allem der 1980er Jahre erfreute und erfreut sich das Modell einer breiten, oft eklektischen Anwendung. Kurz: Stanzel gehört zu den Klassikern der Erzählforschung in deren Gründungsphase während der Nachkriegszeit und in der anschließenden strukturalistischen Phase. Zumindest in einer deutschsprachigen Anthologie erzähltheoretischer Klassiker des 20. Jahrhunderts ist dieser Literaturwissenschaftler eine feste Größe - dokumentiert u.a. in der Anthologie Moderne Erzähltheorie. Grundlagentexte von Henry James bis zur Gegenwart von Karl Wagner (2001, neueste Aufl. 2015), wo Stanzel neben Theodor W. Adorno, Karlheinz Stierle, Wolfgang Iser und Heimito von Doderer den deutschsprachigen Beitrag zur Erzähltheorie im weiteren Sinne repräsentiert.

Was bringt nun der Rückblick des Autors auf die »Erfolgsgeschichte einer Triade« (Untertitel)? Für die Literaturwissenschaft nicht viel Neues; das wenige Neue und das lustvoll variierte Alte sind aber pointiert auf anregende Art und Weise, die vom Verfasser in augenzwinkernden programmatischen Volten ausführlich reflektiert wird:

Dieser Text soll, soweit das möglich ist, dokumentieren, inwiefern sich die kognitiven wie auch assoziativen Funktionen des Gehirns des Nonagenariers des Jahres 2015 von dem des dreißigjährigen Verfassers der Typischen Erzählsituationen von 1955 unterscheiden. Dafür liefern Anlage und Aufbau des Werkes, Auswahl der Themen, Respektierung/ Nichtrespektierung der fachwissenschaftlichen Konventionen, Umfang und Zielrichtung des zeitkritischen Inhalts usw. usw. reichlich Anschauungsmaterial. (S. 13)

Die Leser vom Fach dürfte freilich weniger der respektable Stand der »kognitiven Plastizität « (S. 12) im hohen Alter, wohl aber Diskurs und Habitus eines betagten Literaturwissenschaftlers bei seinem Rückblick auf 
eine wissenschaftliche Karriere interessieren, die von der `Stunde Null $<$ bis zum Internet-Zeitalter reicht. Die Anlage des Buches soll erklärtermaßen jene ‘kreative Entropie` dokumentieren, die sich aus der Fülle der Lebenserfahrung bei gleichzeitigem Wegfall des institutionellen Imperativs zur Einhaltung diskursiver und medialer Grenzen ergeben kann. Insofern ist der gewollt hybride und anekdotische Charakter des Bandes sehr wohl altersbedingt - dokumentiert er doch die Freiheit des Emeritierten zur Missachtung der oben genannten Grenzen, deren Hinnahme und theoretische Reflexion einst Grundvoraussetzung der eigenen akademischen Karriere gewesen sein musste. Der Fokus des Bandes ist ein wissenschaftsbiographischer: zentriert im subjektiven Blick auf die Entstehungs-, Wandlungs- und Wirkungsgeschichte eines theoretischen Bauwerks, das hier greifbar wird als Lebenswerk im wörtlichen Sinne, da die Trennung der sozialen Sphären - private vs. wissenschaftliche Existenz - aufgegeben werden kann. So wird der Band mit Abbildungen von Stanzels »Morschplastik-Skulpturen « aus Kastanienholz illustriert, die Parallelen zur Entstehungsgeschichte des Typenkreises und der Wirkungslogik narrativer Perspektive aufweisen sollen; und die Rezeptionsgeschichte von Stanzels Erzähltheorie wird unter anderem durch das Prisma proto-literarischer Verarbeitung durch den Autor >dokumentiert`, nämlich in Form von Zitat-Collagen (»Found Poems«) aus positiven und negativen Rezensionen.

Die Festschrift kann als konsequente Fortsetzung und Abrundung jenes Ausbauverfahrens gesehen werden, das Stanzel in der jahrzehntelangen Dauerverteidigung und Nachjustierung seiner Erzähltheorie betrieben hat. Die Komposition des Bandes zeichnet die Entstehungs- und Entfaltungsgeschichte dieser Theorie noch einmal nach, wobei die weitgehend bekannten Kontexte, Etappen und Positionen (vgl. zuletzt: Welt als Text. Grundbegriffe der Interpretation, 2011; Verlust einer Jugend. Rückschau eines Neunzigjährigen auf Krieg und Gefangenschaft, 2013) rekapituliert, kommentiert, zusammengefasst oder erweitert werden. Auf die ausgedehnte $>$ Innenperspektive folgt am Ende des Bandes die `Außenperspektive « Gratulationsadressen von FachkollegInnen und die Laudatio zur Verleihung der Ehrendoktorwürde der Universität Marburg (2015).

Der Rückblick auf die biographischen, institutionellen und fachgeschichtlichen Umstände zwischen 1945 und 1962 (Kriegsgefangenschaft in Kanada, Studium in Graz und Harvard, Lehrtätigkeit in Göttingen und Erlangen), sowie auf die literaturwissenschaftlichen und anderen Anreize (R. Wellek, L. Trilling, Kinsey-Report, E. M. Forster, P. Lubbock) für die eigene frühe Theoriebildung, ist ein wertvoller Beitrag zur Wissenschaftsund Ideengeschichte, der einmal mehr die Ergiebigkeit von Transfer- und 
Netzwerkforschung für die Rekonstruktion intellektueller Entwicklungen unter Beweis stellt. Auf die Vorgeschichte folgt die "Erfolgsgeschichte» der Stanzelschen Erzähltheorie, deren Rekapitulation um die wichtigsten Anregungen und Kontroversen gruppiert ist, vom Autor auf den neuesten kritisch-polemischen Stand gebracht. Es geht vor allem, in drei getrennten Kapiteln, um den `berühmt-berüchtigten Typenkreis` (passim, nach einem Urteil der Kritik), die typischen Erzählsituationen in James Joyces Ulysses und das Phänomen der Erlebten Rede; daneben um Einzelfragen wie die Unterscheidung von Perspektivismus und Aperspektivismus oder die typischen Erzählsituationen in der Darstellung von Sex.

Die besondere Dynamik autobiographischer Rückblicke - auch dies eine im Umfeld der `klassischen • Erzähltextanalyse geschärfte Erkenntnis - ergibt sich aus der subjektiv konturierten Differenz zwischen serzählter Zeit` und >Erzählzeit`, zwischen `damals` und `heute` im Spannungsverhältnis von persönlichem Reifungsprozess, lebensweltlichem Wandel und Parametern der narrativen Selbstreflexion. Das dramatische Tempo der lebensweltlichen Veränderungen in der Moderne lässt die Anpassungsleistung des Individuums zur Gretchenfrage, die kulturkritische Klage zum autobiographischen Topos werden. Stanzels Verhältnis zur `erzählten Zeit`, die in der versunkenen Epoche akademischer Vollbeschäftigung, prädigitaler Medien und `klassischer` Narratologie ansetzt, ist aufs Ganze gesehen keineswegs larmoyant; seine Perspektive ist die eines gewitzten und engagierten Beobachters der wissenschaftlichen und lebensweltlichen Entwicklung. Die `Erzählgegenwart` weckt freilich auch die "große Sorge, dass dieses [zeitgenössische] Publikum das Sensorium für die Wahrnehmung der sprachlichen, stilistischen, narrativen Minimalismen [...] angesichts der ständigen massiven Reizüberflutung durch die elektronischen Medien verlieren werde « (S. 154). In welchem Maße die Feststellung eines angeblichen sensorischen Niedergangs einen Reflex auf die Verlagerung der Forschungsinteressen darstellt und somit evtl. auch mit der Skepsis des `Klassikers gegenüber der ` postklassischen Narratologie` und ihrem Anspruch auf gesellschaftskritische und kulturwissenschaftliche Erweiterung der Perspektive zusammenhängt, sei dahingestellt. Eine andere Klage stellt die von der Langzeiterinnerung profitierende Treffsicherheit von Stanzels Zeitkritik eindeutiger unter Beweis: jene über die »Totalanglisierung des anglistischen Diskurses" (S. 155), den Sonderfall einer fächerübergreifenden Entwicklung. - Fazit: Der Grazer Erzählforscher und Imagologe F. K. Stanzel hat ein facettenreiches Einzelporträt des Homo academicus austriacus vorgelegt. 